\title{
EMBEDDING NEAR-RINGS INTO POLYNOMIAL NEAR-RINGS
}

\author{
by JOHN D. P. MELDRUM, GÜNTER PILZ and YONG-SIAN SO*
}

(Received 9th September 1980)

In this paper we will show that large classes of near-rings are embeddable into (or even isomorphic to) near-rings $G^{\vee}[x]$ of polynomials over $G$ in a suitably chosen variety $\mathcal{V}$ of $\Omega$-groups.

For near-rings see [2], for polynomials [1]; for near-rings of polynomials over $\Omega$-groups see [3], [4], [5].

We start with the surprising result that every near-ring is a polynomial near-ring:

Theorem 1. For every near-ring $N$ there is a variety $\mathcal{V}$ of $\Omega$-groups and $a G \in \mathcal{V}$ such that

$$
N \hookrightarrow G[x] .
$$

Proof. By 1.102 of [2], $N$ can be embedded into a near-ring $\bar{N}$ with identity 1 . Now we take $V$ as the subvariety of the variety of all near-rings with identity which is generated by $\bar{N}$ (this last restriction is not necessary for the sequel, but seems to be natural when looking at examples). Now we can consider the map

$$
\phi: \bar{N} \rightarrow \bar{N}^{V}[x]: n \rightarrow n x
$$

$\phi$ easily turns out to be a near-ring homomorphism if $\vec{N}^{\mathcal{V}}[x]$ is considered as a near-ring w.r.t. addition and composition of polynomials. $\phi$ is even a monomorphism (hence an embedding map): take $n \in \operatorname{Ker} \phi$. Then $n x=0$. Suppose that $n \neq 0$. Then the polynomial function $f: \bar{N} \rightarrow \bar{N}$ induced by $n x$ would fulfill $f(1)=n 1=n \neq 0$. Hence $f \neq 0$ (zero function), whence $n x \neq 0$, a contradiction. This proves the theorem.

Now we fix the variety $\mathcal{V}$ and take for $\mathcal{V}$ some important varieties.

We start with the variety $\mathscr{G}$ of groups. Quite unexpectedly, we need information about the idempotents in $G^{g}[x]$. $G[x]$ as an additive group is the free sum of the group $G$ and an infinite cyclic group generated by $x$. The product is composition of functions:

$$
\left(g_{0}+z_{1} x+\ldots+z_{n} x+g_{n}\right) \cdot h=g_{0}+z_{1} h+\ldots+z_{n} h+g_{n}
$$

\footnotetext{
* This study was kindly supported by the Austrian "Fonds zur Förderung der wissenschaftlichen Forschung" (Project Nr. 3479). and by the British Council (Academic link Edinburgh-Linz). The authors give their thanks to these organisations.
} 
where $g_{i} \in G, 0 \leqq i \leqq n, z_{i} \in \mathbb{Z}, 1 \leqq i \leqq n$ and $h \in G[x]$. Obviously, constant elements $g \in G$ and $x$ are idempotent. But that's all:

Theorem 2. The idempotent elements in $G[x]$ ( $G$ a group) are $x$ and the constants.

Proof. Let $e=g_{0}+z_{1} x+\ldots+z_{n} x+g_{n}$ be in reduced (normal) form. $\operatorname{deg}(e)=n$ is the "degree" of $e$. If $n \leqq 1$ then it is fairly easy to see that $e=x$ or $e=c \in G$. So suppose that $n \geqq 2$.

We first remark that if $a$ is an element of a free product of groups written in reduced normal form, then there is a unique element $b$ of maximal length such that $a=$ $-b+c+b$ and this expression on the right is already in reduced form. Then the reduced form of $2 c$ is just $c+c$. Note that $c$ is the cyclically reduced form of $a$. The reduced form of $z a$ is then $-b+z c+b$. In this expression for $a, b$ may be 0 , but $c$ never is.

From the expression for $e$ and the assumptions, we deduce that

$$
e \circ e=g_{0}+z_{1} e+\ldots+z_{n} e+g_{n}
$$

where $g_{0}$ or $g_{n}$ or both or neither may be zero, but no other terms are zero. Let $e=-a+b+a$, with $b$ the cyclically reduced form of $e$.

If $a \neq 0$, then $z_{i} e=-a+z_{i} b+a$ and

$$
e \circ e=g_{0}-a+z_{1} b+a+\ldots+g_{i-1}-a+z_{i} b+a+g_{i}-a+\ldots
$$

The only place where cancellations could start is at $g_{i}$ for some $i$. Also $a$ ends in either $g_{n}$ or $z_{n} x$, so the only cancellation possible is the replacement of $g_{i}$ by $g_{n}+g_{i}-g_{n}$ which is non-zero. There is no cancellation at the ends of the expression for $e \circ e$, except if $g_{0}$ or $g_{n}$ is of order 2 . In any case the degrees considered are not affected. Hence

$$
\operatorname{deg}(e \circ e)=\sum_{i=1}^{n} \operatorname{deg}\left(z_{i} e\right) \geqq 2 \operatorname{deg} e>\operatorname{deg} e
$$

as $n \geqq 2, \operatorname{deg} e=n \geqq 2$ and $\operatorname{deg}\left(z_{i} e\right) \geqq \operatorname{deg} e$ since $\left|z_{i}\right| \geqq 1$. Thus in this case $e \circ e \neq e$.

Now suppose that $a=0$. Then the sum $e+e$ has no cancellations. Consider $e \circ e$ at $g_{i}:$

$$
\ldots+z_{i} e+g_{i}+z_{i+1} e+\ldots
$$

If $\operatorname{sgn} z_{i} \neq \operatorname{sgn} z_{i+1}$, again we have

$$
\pm e+g_{i} \mp e
$$

and, as before, the only possible change is in replacing $g_{i}$ by $g_{n}+g_{i}-g_{n}$ or $-g_{0}+g_{i}+g_{0}$. So assume that $\operatorname{sgn} z_{i}=\operatorname{sgn} z_{i+1}$. Then, at $g_{i}$ we have

or

$$
\ldots+z_{n} x+g_{n}+g_{i}+g_{0}+z_{1} x+\ldots,
$$

$$
\ldots-z_{1} x-g_{0}+g_{i}-g_{n}-z_{n} x+\ldots
$$

If $g_{n}+g_{i}+g_{0}$ or $-g_{0}+g_{i}-g_{n}$ is not zero then there is no more cancellation. If it is zero, then we have

or

$$
2\left(z_{1} x+\ldots+z_{n} x\right)
$$

$$
-2\left(z_{1} x+\ldots+z_{n} x\right)
$$


and the cancellation can not proceed more than halfway along the expression \pm $\left(z_{1} x+\ldots+z_{n} x\right)$. This means that each $g_{i}$ can be considered separately. There is no cancellation at either end since $g_{0} \neq-g_{n}$ apart from replacing $g_{0}$ by $2 g_{0}$ or $g_{0}-g_{n}$, and $g_{n}$ by $2 g_{n}$ or $-g_{0}+g_{n}$. If $g_{0}$ or $g_{n}$ is of order 2 then $2 g_{0}$ or $2 g_{n}$ may be 0 . In any case the degrees considered are not affected. Also $\operatorname{deg}\left( \pm 2\left(z_{1} x+\ldots+z_{n} x\right)\right) \geqq$ $\operatorname{deg}\left(z_{1} x+\ldots+z_{n} x\right)$.

So unless sgn $z_{i}$ is constant for all $i, 1 \leqq i \leqq n,\left|z_{i}\right|=1$ for $1 \leqq i \leqq n$ and $g_{n}+g_{i}+g_{0}=0$ $\left(\operatorname{sgn} z_{i}=+1\right)$ or $-g_{0}+g_{i}-g_{n}=0\left(\operatorname{sgn} z_{i}=-1\right)$ for $1 \leqq i \leqq n-1$, we have

$$
\operatorname{deg}(e \circ e) \geqq 2 \operatorname{deg} e=2 n>\operatorname{deg} e=n,
$$

and hence $e \circ e \neq e$. Otherwise we have

$$
e \circ e=g_{0}+g_{0}+n\left(z_{1} x+\ldots+z_{n} x\right)+g_{n}+g_{n} \quad\left(\operatorname{sgn} z_{i}=+1\right)
$$

or

$$
g_{0}-g_{n}-n\left(z_{1} x+\ldots+z_{n} x\right)-g_{0}+g_{n} \quad\left(\operatorname{sgn} z_{i}=-1\right)
$$

If $e \circ e=e$, then $g_{0}=g_{n}=0$ and so $\operatorname{sgn} z_{1}=\operatorname{sgn} z_{n}$ forces $\operatorname{deg} n\left(z_{1} x+\ldots+z_{n} x\right)=n \operatorname{deg}$ $\left(z_{1} x+\ldots+z_{n} x\right)=n^{2}-(n-1)>n$ as $n \geqq 2$. Thus in all cases it is impossible for $e \circ e=e$ to hold if $n \geqq 2$. This finishes the proof.

Corollary 1. (a) If $e$ is idempotent in $G_{0}[x]$ then $e=0$ or $e=x$.

(b) $G[x]$ has exactly $|G|+1$ idempotents.

Corollary 2. Let $N$ be a non-zero subnear-ring of $G[x]$ with identity $e$. Then $e=x$.

This holds since $e$ is zerosymmetric.

Corollary 3. Let $N$ be a near-ring with identity $e \neq 0$ and let $\phi: N \rightarrow G_{0}[x]$ be an embedding map. Then $\phi(e)=x$.

Corollary 4. Let $\phi$ be as above. If $d \in N(d \neq 0)$ is distributive then there is some $g \in G$ with $\phi(d)=g+x-g$ or $N=\mathbb{Z}$ and $\phi(d)=z x, z \in \mathbb{Z}$.

Proof. $d$ is zero-symmetric, hence $\phi(d)$ has the form

Now

$$
\phi(d)=\sum_{i=1}^{n}\left(g_{i}+z_{i} x-g_{i}\right) \quad\left(g_{i} \in G, z_{i} \in \mathbb{Z}, g_{i} \neq g_{i+1}\right)
$$

$\phi(d) \circ(x+x)=\phi(d) \circ \phi(e+e)=\phi(d \circ(e+e))=\phi(d \circ e+d \circ e)=\phi(d+d)=\phi(d)+\phi(d)$.

Hence

$$
\sum_{i=1}^{n}\left(g_{i}+2 z_{i} x-g_{i}\right)=\sum_{i=1}^{n}\left(g_{i}+z_{i} x-g_{i}\right)+\sum_{i=1}^{n}\left(g_{i}+z_{i} x-g_{i}\right)
$$

However, this relation can only hold if the right hand side collapses, i.e. if $g_{n}=g_{1}, z_{n}=$ $-z_{1}, g_{n-1}=g_{2}$, and so on. So the right side is either zero, whence $g_{1}+2 z_{1}-g_{1}+\ldots+$ $g_{n}+2 z_{n} x-g_{n}=0$, a contradiction, or $n=1$ in which case $\phi(d)=g_{1}+z_{1} x-g_{1}$.

Computing $\phi(d \circ(e+d))$ in two ways and comparing the results we obtain $z_{1}=1$ or $g_{1}=0$. If $z_{1}=1$, we have $\phi(d)=g+x-g$. If $g_{1}=0$, then $\phi(d)=z_{1} x$. Suppose $z_{1} \neq 1$ and 
$\phi(N) \supset\{z x ; z \in \mathbb{Z}\}$. Then $z_{1} x \circ(a+b)=z_{1}(a+b), \quad z_{1} x \circ a+z_{1} x \circ b=z_{1} a+z_{1} b$. So $z_{1}(a+b)=z_{1} a+z_{1} b$. Take one of the pair $a, b$ to be $x$, the other to lie in $\phi(N)$, but not of the form $z x, z \in \mathbb{Z}$. By examining the various cases that can arise it is easy to see that we get a contradiction. Thus $N=\mathbb{Z}$ and $\phi(N)=\{z x ; z \in \mathbb{Z}\}$.

Now we prove one of the few existing results that certain embeddings can not take place.

Theorem 3. There exist distributively generated near-rings which cannot be embedded into some $G[x]$.

Proof. Let $N$ be a distributively generated near-ring such that $(N,+)$ is not a free group (for instance, take $N$ finite, but $\neq\{0\}$ ). Let $\phi$ be a monomorphism of $N$ into some $G[x]$. Then $\phi(N) \subseteq G_{0}[x]$. But $\left(G_{0}[x],+\right)$ is a free group and hence so is $(\phi(N),+)$. As $(\phi(N),+) \cong(N,+)$ we have a contradiction.

Consequently, not every generalized distributively generated (g.d.g.) near-ring can be embedded into some $G[x]$. Here, a near-ring $N$ is g.d.g. if $N$ is (additively) generated by distributive and constant elements (see [5]). $G[x]$ itself is g.d.g.

On the other hand we have shown in [5] that every finite near-ring can be embedded into the g.d.g. near-ring $P(G)$ of all polynomial functions on a suitable finite, simple, non-abelian group $G$.

We now consider the embedding of $G[x]$ in $P(H)$ for a suitable group $H$. To do this we need some information on the structure of $G[x]$. From [1], each element of $G[x]$ can be written uniquely in the form

$$
g_{1}+z_{1} x-g_{1}+\ldots+g_{n}+z_{n} x-g_{n}+h=\sum_{i=1}^{n}\left(g_{i}+x-g_{i}\right)+h
$$

where each $g_{i}+x-g_{i}$ is distributive and $h$ is a constant element. It is immediate that

$$
\mathrm{Gp}\langle g+x-g ; g \in G\rangle=G_{0}[x],
$$

is the free d.g. near-ring on the set,

$$
\{g+x-g ; g \in G\}
$$

of distributive elements. Also

$$
\left(g_{1}+x-g_{1}\right) \circ\left(g_{2}+x-g_{2}\right)=g_{1}+g_{2}+x-g_{2}-g_{1} .
$$

So the multiplicative set of distributive elements $\{g+x-g ; g \in G\}$ is a group which is isomorphic as an abstract group to the additive group $(G,+)$ under the isomorphism

$$
g+x-g \rightarrow g
$$

As an additive group $(G[x],+)$ has $\left(G_{0}[x],+\right)$ as a normal subgroup and $G[x]=$ $G_{0}[x]+G_{c}[x]$ where $G_{c}[x]$ is the constant subnear-ring isomorphic as an additive group to $\mathrm{G}$. Also, from the normal form given above, if $h \in G_{c}[x]$,

$$
h+(g+x-g)-h=(h+g)+x-(h+g) \text {. }
$$


So the automorphism induced in $\left(G_{0}[x],+\right)$ by conjugation by an element $h \in G_{c}[x]$ maps

$$
g+x-g \rightarrow(h+g)+x-(h+g)
$$

The automorphisms induced in $\left(G_{0}[x],+\right)$ by the elements of $G_{c}[x] \cong G$ is that induced in the free group $\left(G_{0}[x],+\right)$ by the permutation of the free generators of $G_{0}[x]$ by the left regular representation.

This determines the additive structure of $G[x]$ completely. Now we consider the multiplicative structure. Since the right distributive law is satisfied, we only need to consider products of the form $x y$ where $y$ is a general element and $x$ is either an element of the form $g+x-g$ or $h$. But $h \circ y=h$ for all $y \in G[x]$. So consider

$$
\begin{gathered}
(g+x-g) \circ\left(\sum_{i=1}^{n} g_{i}+x-g_{i}+h\right)=g+\sum_{i=1}^{n} g_{i}+x-g_{i}+h-g \\
=\sum_{i=1}^{n}\left(g+g_{i}+x-g_{i}-g\right)+g+h-g .
\end{gathered}
$$

We now turn to the construction of the group $H$ which we will need. Let $G^{*}=$ $\left\{g^{*} ; g \in G\right\}$ be a set in one-one correspondence with the elements of the group $G$ and define $K$ to be the free group on the set $G^{*}$. Let $\tau: G \rightarrow$ Aut $K$ be defined by $\tau(g)\left(g_{1}^{*}\right)=\left(g+g_{1}\right)^{*}$ for all $g_{1} \in K$. This is enough to define $\tau(g) \in$ Aut $K$ since $K$ is a free group on $G^{*}$. Define $H$ as the semidirect product of $K$ by $G$ using $\tau$. Consider $P(H)$ and note that $P_{0}(H) \cong I(H)$, the near-ring generated by $\operatorname{Inn}(H)$, the inner automorphisms of $H$ and $P_{c}(H) \cong H$. Let the constant map which sends $H$ to $h \in H$ be denoted by $\theta(h)$.

Without loss of generality, assume that $G \subseteq H$. Consider the subnear-ring $N$ of $P(H)$ generated by $\tau(G)$ and $\theta(G)$, where $\tau(G)$ are the inner automorphisms of $H$ determined by $G$. We first show that $\operatorname{Gp}\langle\tau(G)\rangle$ is a free group on $\tau(G)$.

Consider $\sum_{i=1}^{n} \varepsilon_{i} \tau\left(g_{i}\right)$ where $\varepsilon_{i}= \pm 1,1 \leqq i \leqq n$. Then

$$
\begin{aligned}
\left(\sum_{i=1}^{n} \varepsilon_{i} \tau\left(g_{i}\right)\right)\left(0^{*}\right) & =\sum_{i=1}^{n} \varepsilon_{i} \tau\left(g_{i}\right)\left(0^{*}\right) \\
& =\sum_{i=1}^{n} \varepsilon_{i}\left(g_{i}+0\right)^{*} \\
& =\sum_{i=1}^{n} \varepsilon_{i} g_{i}^{*} .
\end{aligned}
$$

and this is not 0 unless $\sum_{i=1}^{n} \varepsilon_{i} \tau\left(g_{i}\right)$ is the trivial word since $K$ is free on $G^{*}$. Hence $\sum_{i=1}^{n} \varepsilon_{i} \tau\left(g_{i}\right) \neq 0$ in $P(H)$. It is trivial to check that $\tau\left(g_{1}\right) \circ \tau\left(g_{2}\right)=\tau\left(g_{1}+g_{2}\right)$. Also $\tau(g) 0^{*}=$ $g^{*} \neq g_{1}^{*}=\tau\left(g_{1}\right) 0^{*}$ if $g \neq g_{1}$.

Thus $\tau(G)$ is a multiplicative group isomorphic to the additive group $G$ and $\mathrm{Gp}\langle\tau(G)\rangle$ is the free d.g. near-ring on $\tau(G)$. Call this near-ring $N_{0}$. Then $N_{0} \cong G_{0}[x]$, with $\tau(g) \rightarrow g+x-g$. Again it is trivial that $\theta(g)+\tau\left(g_{i}\right)-\theta(g)=\tau\left(g+g_{i}\right)$. Hence 
$(N,+) \cong(G[x],+)$ and $N_{c} \cong G_{c}[x], N_{0} \cong G_{0}[x]$. Finally consider $\tau(g) \circ\left(\sum_{i=1}^{n} \tau\left(g_{i}\right)+\theta(k)\right)$ for $k \in G$. Let $h \in H$.

$$
\begin{aligned}
\tau(g) \circ\left(\sum_{i=1}^{n} \tau\left(g_{i}\right)+\theta(k)\right)(h) & =\tau(g)\left(\sum_{i=1}^{n} \tau\left(g_{i}\right)(h)+k\right) \\
& =\sum_{i=1}^{n} \tau\left(g+g_{i}\right)(h)+g+k-g=\left(\sum_{i=1}^{n} \tau\left(g+g_{i}\right)+\theta(g+k-g)\right)(h) .
\end{aligned}
$$

This is enough to complete the proof of the following theorem.

Theorem 4. Let $G$ be a group. Then there exists a group $H$ such that $G[x]$ can be embedded in $P(H)$.

Corollary 5. Let $G$ be a group. Then there exists a group $H$ such that $G_{0}[x]$ can be embedded in $P_{0}(H)$.

Note that $P_{0}(H) \cong I(H)$ and that the group $H$ is isomorphic as a group to $(G[x],+)$ in the construction given above.

Now we change the variety of groups to $\mathscr{A}$, the variety of abelian groups. If $A \in \mathscr{A}$ then $A] x\left[:=A^{\mathscr{A}}[x]=\{a+z x \mid a \in A, z \in \mathbb{Z}\}\right.$ is an abstract affine near-ring.

If we look at the variety $\mathcal{M}_{R}$ of $R$-modules over a ring $R$ and $M \in M_{R}$ then $M_{R}[x]:=M^{M_{R}}[x]=\{m+r x \mid m \in M, r \in R\}$ is abstract affine, too.

Hence it is natural to ask which abstract affine near-rings can be embedded into $A] x[$ or $\left.M_{R}[x] . A\right] x\left[\right.$ does not suffice, but $M_{R}[x]$ does an excellent job:

Theorem 5. (i) Not every abstract affine near-ring $N$ can be embedded into some $A] x[(A$ an abelian group $)$.

(ii) Every abstract affine near-ring is isomorphic to a suitably chosen $M_{R}[x]\left(M_{R}\right.$ an $R$-module).

Proof. (i) We take $N:=\mathbb{R} \times \mathbb{R}$ with + componentwise and $(r, s) \cdot\left(r^{\prime}, s^{\prime}\right):=$ $\left(r+s r^{\prime}, s s^{\prime}\right)$. Then $N$ is an abstract affine near-ring. Suppose that $A$ is an abelian group with $N \hookrightarrow A] x\left[\right.$ by some monomorphism $\phi . N_{0}=\{0\} \times \mathbb{R}$ will then be embedded into $(A] x[)_{0} \cong \mathbb{Z}$, a contradiction.

(ii) Let $N$ be an abstract affine near-ring. Then $N_{0}$ is a ring and the constants $N_{c}$ are an $N_{0}$-module. We consider the map $\psi: N \rightarrow\left(N_{c}\right)_{\left(N_{0}\right)}[x]: n_{c}+n_{0} \rightarrow n_{c}+n_{0} x . \psi$ is obviously a group isomorphism. Also, for all $n, n^{\prime} \in N, n=n_{c}+n_{0}, n^{\prime}=n_{c}^{\prime}+n_{0}^{\prime}$, we get

$$
\psi\left(n n^{\prime}\right)=\psi\left(\left(n_{c}+n_{0}\right)\left(n_{c}^{\prime}+n_{0}^{\prime}\right)\right)=\psi\left(n_{c}+n_{0} n_{c}^{\prime}+n_{0} n_{0}^{\prime}\right) .
$$

Since $n_{c}+n_{0} n_{c}^{\prime} \in N_{c}$ and $n_{0} n_{0}^{\prime} \in N_{0}$, this expression equals

$$
\left(n_{c}+n_{0} n_{c}^{\prime}\right)+\left(n_{0} n_{0}^{\prime}\right) x=\left(n_{c}+n_{0} x\right) \circ\left(n_{c}^{\prime}+n_{0}^{\prime} x\right)=\psi(n) \circ \psi\left(n^{\prime}\right) .
$$

Hence we get $N \cong\left(N_{c}\right)_{\left(N_{0}\right)}[x]$. 
The authors hope that these results will help to solve the long-standing problem whether or not every zerosymmetric near-ring can be embedded into a d.g. near-ring (see [2], p. 178).

\section{REFERENCES}

1. H. LausCh, and W. NöBauer, Algebra of polynomials (North-Holland, Amsterdam, 1973).

2. G. Priz, Near-rings, (North-Holland, Amsterdam, 1977).

3. G.Puz and Y. S. So, Near-rings of polynomials and polynomial functions, J. Austral.Math. Soc. (Series A) 29 (1980), 61-70.

4. G. PILZ and Y. S. So, Near-rings of polynomials over $\Omega$-groups, submitted.

5. G. PILz and Y. S. So, Generalized distributively generated near-rings, submitted.

Dept. of Mathematics

UNIV. OF EDINBURGH

MAYFIELD RD

EDINBURGH EH9 3JZ

U.K.

INSTITUT FÜR MATHEMATIK

UNIVERSITÄT LINZ

A-4040 Linz

Austria 